\title{
Certain Analytic Functions with Missing Coefficients
}

\author{
Cai-Mei Yan $^{1 *}$ and Jin-Lin Liu ${ }^{2}$ \\ ${ }^{1}$ Information Engineering College, Yangzhou University, Yangzhou, 225002, P.R. China \\ Email: cmyan@yzu.edu.cn \\ ${ }^{2}$ Department of Mathematics, Yangzhou University, Yangzhou, 225002, P.R. China \\ Email: jlliu@yzu.edu.cn
}

\begin{abstract}
Let $\mathcal{A}_{n}$ denote the class of functions of the form $f(z)=z+\sum_{k=n+1}^{\infty} a_{k} z^{k}$, which are analytic in the open unit disk $U=\{z:|z|<1\}$. In this note we shall find $\max _{|z|=r<1} \operatorname{Re}\left\{f^{\prime}(z)+\right.$ $\left.\alpha z f^{\prime \prime}(z)\right\}$ under the condition $f^{\prime}(z) \prec \frac{1+A z}{1+B z}$ for $f \in \mathcal{A}_{n}$.
\end{abstract}

Keywords: Analytic function, subordination, missing coefficient.

\section{Introduction}

Throughout our present investigation, we assume that

$$
n \in N,-1 \leq B<1, B<A, \alpha>0 \text { and } \beta<1 \text {. }
$$

Let $\mathcal{A}_{n}$ denote the class of functions of the form:

$$
f(z)=z+\sum_{k=n+1}^{\infty} a_{k} z^{k}
$$

which are analytic in the open unit disk $U=\{z:|z|<1\}$.

For functions $f$ and $g$ analytic in $U$, we say that $f$ is subordinate to $g$ and write $f(z) \prec g(z) \quad(z \in U)$, if there exists an analytic function $w(z)$ in $U$ such that

$$
|w(z)| \leq|z| \quad \text { and } \quad f(z)=g(w(z)) \quad(z \in U)
$$

Furthermore, if the function $g$ is univalent in $U$, then

$$
f(z) \prec g(z) \quad(z \in U) \Longleftrightarrow f(0)=g(0) \quad \text { and } \quad f(U) \subset g(U) .
$$

In a recent paper [3], Gao and Zhou considered the following subclass of $\mathcal{A}_{1}$ :

$$
R(\beta, \alpha)=\left\{f \in \mathcal{A}_{1}: \operatorname{Re}\left\{f^{\prime}(z)+\alpha z f^{\prime \prime}(z)\right\}>\beta \quad(z \in U)\right\} .
$$

Some interesting properties of the class $R(\beta, \alpha)$ have been given in [1]. For further information of the class $R(\beta, \alpha)$ one can see the related papers (see, e.g., [234561789]). Inspired by the above works, in this note we shall find

$$
\max _{|z|=r<1} \operatorname{Re}\left\{f^{\prime}(z)+\alpha z f^{\prime \prime}(z)\right\}
$$

under the condition $f^{\prime}(z) \prec \frac{1+A z}{1+B z}$.

\section{Main Results}

Theorem 2.1. Let $f$ belong to the class $\mathcal{A}_{n}$ and satisfy

$$
f^{\prime}(z) \prec \frac{1+A z}{1+B z} \quad(z \in U) .
$$


Then

$$
\operatorname{Re}\left\{f^{\prime}(z)+\alpha z f^{\prime \prime}(z)\right\} \leq \frac{1+(A+B+n \alpha(A-B)) r^{n}+A B r^{2 n}}{\left(1+B r^{n}\right)^{2}} \text { if } M_{n}(A, B, \alpha, r) \leq 0,
$$

or

$$
\operatorname{Re}\left\{f^{\prime}(z)+\alpha z f^{\prime \prime}(z)\right\} \leq \frac{L_{n}^{2}-4 \alpha^{2} K_{A} K_{B}}{4 \alpha(A-B) r^{n-1}\left(1-r^{2}\right) K_{B}} \text { if } M_{n}(A, B, \alpha, r) \geq 0
$$

where

$$
\left\{\begin{array}{l}
K_{A}=1-A^{2} r^{2 n}+n A r^{n-1}\left(1-r^{2}\right) \\
K_{B}=1-B^{2} r^{2 n}+n B r^{n-1}\left(1-r^{2}\right) \\
L_{n}=2 \alpha\left(1-A B r^{2 n}\right)+n \alpha(A+B) r^{n-1}\left(1-r^{2}\right)+(A-B) r^{n-1}\left(1-r^{2}\right) \\
M_{n}(A, B, \alpha, r)=2 \alpha K_{B}\left(1+A r^{n}\right)-L_{n}\left(1+B r^{n}\right) .
\end{array}\right.
$$

The result is sharp.

Proof. Equality in (2.2) occurs for $z=0$. Thus we assume that $0<|z|=r<1$. From (2.1) we can write

$$
f^{\prime}(z)=\frac{1+A z^{n} \varphi(z)}{1+B z^{n} \varphi(z)} \quad(z \in U)
$$

where $\varphi(z)$ is analytic and $|\varphi(z)| \leq 1$ in $U$. It follows from (2.5) that

$$
\begin{aligned}
f^{\prime}(z)+\alpha z f^{\prime \prime}(z) & =f^{\prime}(z)+\frac{\alpha(A-B) z^{n}\left(n \varphi(z)+z \varphi^{\prime}(z)\right)}{\left(1+B z^{n} \varphi(z)\right)^{2}} \\
& =f^{\prime}(z)+\frac{n \alpha}{A-B}\left(A-B f^{\prime}(z)\right)\left(f^{\prime}(z)-1\right)+\frac{\alpha(A-B) z^{n+1} \varphi^{\prime}(z)}{\left(1+B z^{n} \varphi(z)\right)^{2}} .
\end{aligned}
$$

With the help of the Carathéodory inequality:

$$
\left|\varphi^{\prime}(z)\right| \leq \frac{1-|\varphi(z)|^{2}}{1-r^{2}}
$$

we obtain

$$
\begin{aligned}
\operatorname{Re}\left\{\frac{z^{n+1} \varphi^{\prime}(z)}{\left(1+B z^{n} \varphi(z)\right)^{2}}\right\} & \leq \frac{r^{n+1}\left(1-|\varphi(z)|^{2}\right)}{\left(1-r^{2}\right)\left|1+B z^{n} \varphi(z)\right|^{2}} \\
& =\frac{r^{2 n}\left|A-B f^{\prime}(z)\right|^{2}-\left|f^{\prime}(z)-1\right|^{2}}{(A-B)^{2} r^{n-1}\left(1-r^{2}\right)} .
\end{aligned}
$$

Put $f^{\prime}(z)=u+i v \quad(u, v \in R)$. Then (2.6) and (2.7) provide

$$
\begin{aligned}
\operatorname{Re}\left\{f^{\prime}(z)+\alpha z f^{\prime \prime}(z)\right\} \leq & \left(1+n \alpha \frac{A+B}{A-B}\right) u-\frac{n \alpha A}{A-B}-\frac{n \alpha B}{A-B}\left(u^{2}-v^{2}\right) \\
& +\alpha \frac{r^{2 n}\left((A-B u)^{2}+(B v)^{2}\right)-\left((u-1)^{2}+v^{2}\right)}{(A-B) r^{n-1}\left(1-r^{2}\right)} \\
= & \left(1+n \alpha \frac{A+B}{A-B}\right) u-\frac{n \alpha}{A-B}\left(A+B u^{2}\right)+\alpha \frac{r^{2 n}(A-B u)^{2}-(u-1)^{2}}{(A-B) r^{n-1}\left(1-r^{2}\right)} \\
& +\frac{\alpha}{A-B}\left(n B-\frac{1-B^{2} r^{2 n}}{r^{n-1}\left(1-r^{2}\right)}\right) v^{2} .
\end{aligned}
$$

Note that

$$
\begin{aligned}
\frac{1-B^{2} r^{2 n}}{r^{n-1}\left(1-r^{2}\right)} & \geq \frac{1-r^{2 n}}{r^{n-1}\left(1-r^{2}\right)}=\frac{1}{r^{n-1}}\left(1+r^{2}+r^{4}+\cdots+r^{2(n-2)}+r^{2(n-1)}\right) \\
& =\frac{1}{2 r^{n-1}}\left[\left(1+r^{2(n-1)}\right)+\left(r^{2}+r^{2(n-2)}\right)+\cdots+\left(r^{2(n-1)}+1\right)\right] \\
& \geq n \geq n B .
\end{aligned}
$$


Combining (2.8) and (2.9) we get

$$
\begin{aligned}
\operatorname{Re}\left\{f^{\prime}(z)+\alpha z f^{\prime \prime}(z)\right\} & \leq\left(1+n \alpha \frac{A+B}{A-B}\right) u-\frac{n \alpha}{A-B}\left(A+B u^{2}\right)+\alpha \frac{r^{2 n}(A-B u)^{2}-(u-1)^{2}}{(A-B) r^{n-1}\left(1-r^{2}\right)} \\
& =\psi_{n}(u) \quad \text { (say). }
\end{aligned}
$$

It is well known that for $|\xi| \leq \sigma \quad(\sigma<1)$,

$$
\left|\frac{1+A \xi}{1+B \xi}-\frac{1-A B \sigma^{2}}{1-B^{2} \sigma^{2}}\right| \leq \frac{(A-B) \sigma}{1-B^{2} \sigma^{2}}
$$

and

$$
\frac{1-A \sigma}{1-B \sigma} \leq \operatorname{Re}\left\{\frac{1+A \xi}{1+B \xi}\right\} \leq \frac{1+A \sigma}{1+B \sigma}
$$

Also (2.5) and (2.12) imply that

$$
\frac{1-A r^{n}}{1-B r^{n}} \leq \operatorname{Re}\left\{f^{\prime}(z)\right\} \leq \frac{1+A r^{n}}{1+B r^{n}}
$$

Let us now calculate the maximum value of $\psi_{n}(u)$ on the segment $\left[\frac{1-A r^{n}}{1-B r^{n}}, \frac{1+A r^{n}}{1+B r^{n}}\right]$. Obviously,

$$
\begin{gathered}
\psi_{n}^{\prime}(u)=1+n \alpha \frac{A+B}{A-B}-\frac{2 n \alpha B}{A-B} u+2 \alpha \frac{\left(1-A B r^{2 n}\right)-\left(1-B^{2} r^{2 n}\right) u}{(A-B) r^{n-1}\left(1-r^{2}\right)}, \\
\psi_{n}^{\prime \prime}(u)=-\frac{2 \alpha}{A-B}\left(n B+\frac{1-B^{2} r^{2 n}}{r^{n-1}\left(1-r^{2}\right)}\right)<0 \quad(\text { see }(2.9))
\end{gathered}
$$

and $\psi_{n}^{\prime}(u)=0$ if and only if

$$
\begin{aligned}
u=u_{n}= & \frac{2 \alpha\left(1-A B r^{2 n}\right)+n \alpha(A+B) r^{n-1}\left(1-r^{2}\right)+(A-B) r^{n-1}\left(1-r^{2}\right)}{2 \alpha\left[1-B^{2} r^{2 n}+n B r^{n-1}\left(1-r^{2}\right)\right]} \\
& =\frac{L_{n}}{2 \alpha K_{B}} \quad(\operatorname{see}(2.4)) .
\end{aligned}
$$

Since

$$
\begin{aligned}
& 2 \alpha K_{B}\left(1-A r^{n}\right)-L_{n}\left(1-B r^{n}\right) \\
& \quad=2 \alpha\left[\left(1-A r^{n}\right)\left(1-B^{2} r^{2 n}\right)-\left(1-B r^{n}\right)\left(1-A B r^{2 n}\right)\right] \\
& \quad-n \alpha r^{n-1}\left(1-r^{2}\right)\left[(A+B)\left(1-B r^{n}\right)-2 B\left(1-A r^{n}\right)\right]-(A-B) r^{n-1}\left(1-r^{2}\right)\left(1-B r^{n}\right) \\
& \quad=-2 \alpha(A-B) r^{n}\left(1-B r^{n}\right)-n \alpha(A-B) r^{n-1}\left(1-r^{2}\right)\left(1+B r^{n}\right)-(A-B) r^{n-1}\left(1-r^{2}\right)\left(1-B r^{n}\right) \\
& \quad<0
\end{aligned}
$$

we see that

$$
u_{n}>\frac{1-A r^{n}}{1-B r^{n}}
$$

But $u_{n}$ is not always less than $\frac{1+A r^{n}}{1+B r^{n}}$. The following two cases arise.

Case (i). $u_{n} \geq \frac{1+A r^{n}}{1+B r^{n}}$, that is, $M_{n}(A, B, \alpha, r)$ (given by $\left.(2.4)\right) \leq 0$. In view of $\psi_{n}^{\prime}\left(u_{n}\right)=0$ and $(2.13)$, the function $\psi_{n}(u)$ is increasing on the segment $\left[\frac{1-A r^{n}}{1-B r^{n}}, \frac{1+A r^{n}}{1+B r^{n}}\right]$. Therefore we deduce from $(2.10)$ that, 
if $M_{n}(A, B, \alpha, r) \leq 0$, then

$$
\begin{aligned}
\operatorname{Re}\left\{f^{\prime}(z)+\alpha z f^{\prime \prime}(z)\right\} & \leq \psi_{n}\left(\frac{1+A r^{n}}{1+B r^{n}}\right) \\
& =\left(1+n \alpha \frac{A+B}{A-B}\right)\left(\frac{1+A r^{n}}{1+B r^{n}}\right)-\frac{n \alpha}{A-B}\left(A+B\left(\frac{1+A r^{n}}{1+B r^{n}}\right)^{2}\right) \\
& =\frac{1+A r^{n}}{1+B r^{n}}-\frac{n \alpha}{A-B}\left(1-\frac{1+A r^{n}}{1+B r^{n}}\right)\left(A-B \frac{1+A r^{n}}{1+B r^{n}}\right) \\
& =\frac{1+(A+B+n \alpha(A-B)) r^{n}+A B r^{2 n}}{\left(1+B r^{n}\right)^{2}} .
\end{aligned}
$$

This proves (2.2).

Next we consider the function $f$ defined by

$$
f(z)=\int_{0}^{z} \frac{1+A t^{n}}{1+B t^{n}} \mathrm{~d} t
$$

which satisfies the condition (2.1). It is easy to check that

$$
f^{\prime}(r)+\alpha r f^{\prime \prime}(r)=\frac{1+(A+B+n \alpha(A-B)) r^{n}+A B r^{2 n}}{\left(1+B r^{n}\right)^{2}},
$$

which shows that the inequality (2.2) is sharp.

Case (ii). $u_{n} \leq \frac{1+A r^{n}}{1+B r^{n}}$, that is, $M_{n}(A, B, \alpha, r) \geq 0$. In this case we easily have

$$
\operatorname{Re}\left\{f^{\prime}(z)+\alpha z f^{\prime \prime}(z)\right\} \leq \psi_{n}\left(u_{n}\right) .
$$

In view of $(2.4), \psi_{n}(u)$ in $(2.10)$ can be written as

$$
\psi_{n}(u)=\frac{-\alpha K_{B} u^{2}+L_{n} u-\alpha K_{A}}{(A-B) r^{n-1}\left(1-r^{2}\right)} .
$$

Therefore, if $M_{n}(A, B, \alpha, r) \geq 0$, then it follows from (2.14), (2.16) and (2.17) that

$$
\begin{aligned}
\operatorname{Re}\left\{f^{\prime}(z)+\alpha z f^{\prime \prime}(z)\right\} & \leq \frac{-\alpha K_{B} u_{n}^{2}+L_{n} u_{n}-\alpha K_{A}}{(A-B) r^{n-1}\left(1-r^{2}\right)} \\
& =\frac{L_{n}^{2}-4 \alpha^{2} K_{A} K_{B}}{4 \alpha(A-B) r^{n-1}\left(1-r^{2}\right) K_{B}} .
\end{aligned}
$$

To show that the inequality (2.3) is sharp, we take

$$
f(z)=\int_{0}^{z} \frac{1+A t^{n} \varphi(t)}{1+B t^{n} \varphi(t)} \mathrm{d} t \quad \text { and } \quad \varphi(z)=\frac{z-c_{n}}{1-c_{n} z}
$$

where $c_{n} \in R$ is determined by

$$
f^{\prime}(r)=\frac{1+A r^{n} \varphi(r)}{1+B r^{n} \varphi(r)}=u_{n} \in\left(\frac{1-A r^{n}}{1-B r^{n}}, \frac{1+A r^{n}}{1+B r^{n}}\right] .
$$

Clearly, $-1<\varphi(r) \leq 1,-1 \leq c_{n}<1,|\varphi(z)| \leq 1(z \in U)$, and so $f$ satisfies the condition (2.1). Since

$$
\varphi^{\prime}(r)=\frac{1-c_{n}^{2}}{\left(1-c_{n} r\right)^{2}}=\frac{1-|\varphi(r)|^{2}}{1-r^{2}},
$$

from the above argument we find that

$$
f^{\prime}(r)+\alpha r f^{\prime \prime}(r)=\psi_{n}\left(u_{n}\right) .
$$

Now the proof of the theorem is completed. 
Corollary 2.2. Let $f$ belong to the class $\mathcal{A}_{1}$ and satisfy $\operatorname{Re}\left\{f^{\prime}(z)\right\}>\beta(\beta<1 ; z \in U)$. Then for $|z|=r<1$,

$$
\operatorname{Re}\left\{f^{\prime}(z)+\alpha z f^{\prime \prime}(z)\right\} \leq \beta+(1-\beta) \frac{1+2 \alpha r-r^{2}}{(1-r)^{2}} .
$$

The result is sharp.

Proof. By considering $\frac{f^{\prime}(z)-\beta}{1-\beta}$ instead of $f^{\prime}(z)$, we only need to prove the corollary for $\beta=0$. Setting $n=A=1$ and $B=-1$ in (2.4), we get

$$
K_{1}=2\left(1-r^{2}\right), \quad K_{-1}=0, \quad L_{1}=2 \alpha\left(1+r^{2}\right)+2\left(1-r^{2}\right)
$$

and

$$
M_{1}(1,-1, \alpha, r)=-2(1-r)\left[1+\alpha-(1-\alpha) r^{2}\right] \leq 0 .
$$

Consequently, an application of (2.2) in Theorem 2.1 yields

$$
\operatorname{Re}\left\{f^{\prime}(z)+\alpha z f^{\prime \prime}(z)\right\} \leq \frac{1+2 \alpha r-r^{2}}{(1-r)^{2}} .
$$

Furthermore the sharpness follows immediately from that of Theorem 2.1.

Acknowledgments. The authors would like to express sincere thanks to the referee for careful reading and suggestions which helped us to improve the paper. This work is supported by National Natural Science Foundation of China(Grant No.11571299) and Natural Science Foundation of Jiangsu Province(Grant No. BK20151304).

\section{References}

1. C.-Y. Gao and S.-Q. Zhou, "Certain subclass of starlike functions," Appl. Math. Comput., vol. 187, pp. 176-182, 2007.

2. R. M. Ali, "On a subclass of starlike functions," Rocky Mount. J. Math., vol. 24, pp. 447-451, 1994.

3. P. N. Chichra, "New subclasses of the class of close-to-convex functions," Proc. Amer. Math. Soc., vol. 62, pp. 37-43, 1977.

4. W. Janowski, "Some extremal problems for certain families of analytic functions," Ann. Polon. Math., vol. 28, pp. 297-326, 1973.

5. H. Silverman, "A class of bounded starlike functions," Int. J. Math. Math. Sci., vol. 17, pp. 249-252, 1994.

6. R. Singh and S. Singh, "Convolution properties of a class of starlike functions," Proc. Amer. Math. Soc., vol. 106, pp. 145-152, 1989.

7. D.-G. Y. H. M. Srivastava and N.-E. Xu, "Some subclasses of meromorphically multivalent functions associated with a linear operator," Appl. Math. Comput., vol. 195, pp. 11-23, 2008.

8. M. C. H. M. Srivastava, S. Bulut and N. Yagmur, "Coefficient estimates for a general subclass of analytic and bi-univalent functions," Filomat, vol. 27, no. 5, pp. 831-842, 2013.

9. G. I. O. H. M. Srivastava, J. K. Prajapat and R. Sendrutiu, "Geometric properties of a certain general family of integral operators," Filomat, vol. 28, no. 4, pp. 745-754, 2014. 